\title{
Status Report on Silicon Photomultiplier Development and its Applications $^{\#}$
}

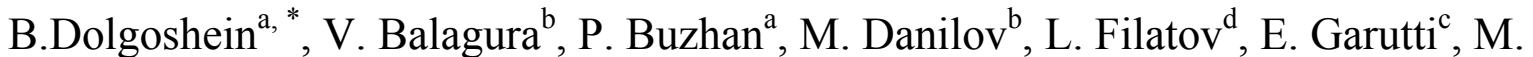

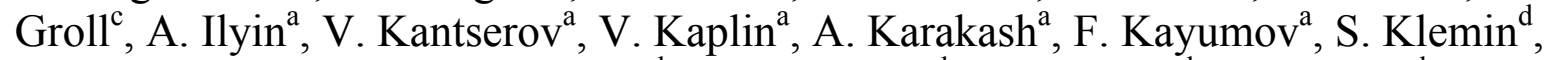
V. Korbel ${ }^{c}$, H. Meyer ${ }^{c}$, R. Mizuk ${ }^{b}$, V. Morgunov ${ }^{b}$, E. Novikov ${ }^{b}$, P. Pakhlov , E. Popova $^{\mathrm{a}}$, V. Rusinov ${ }^{\mathrm{b}}$, F. Sefkow ${ }^{\mathrm{c}}$, E. Tarkovsky ${ }^{\mathrm{b}}$, I. Tikhomirov ${ }^{\mathrm{b}}$

Calice/SiPM Collaboration

${ }^{a}$ Moscow Engineering and Physics Institute, Kashirskoe shosse 31,115409,Moscow,Russia

${ }^{b}$ Institute of Theoretical and Experimental Physics, B. Cheremushkinskaya 25, Moscow, Russia

${ }^{c}$ DESY, Notkestrasse 85, 22607 Hamburg, Germany

${ }^{c}$ State Unitary Science \& Production Enterprise (PULSAR), Okruzhnoy proezd 27, Moscow, Russia

Elsevier use only: Received date here; revised date here; accepted date here

\begin{abstract}
The state of art of the Silicon Photomultipliers (SiPM's) - their features, possibilities and applications - is given. The significant progress of this novel technique of photo detection is described and discussed.

(C) 2001 Elsevier Science. All rights reserved

Keywords: Silicon Photomultiplier, hadron calorimeter, wavelength shifter, scintillation detector, time of flight measurements PACS: the PACS codes can be found at the home page of NIMA (left column, under Contents Services):
\end{abstract}

\footnotetext{
\# Presented by B.Dolgoshein at TRD2005 conference, Bari, Italy, 7.-10. September 2005

* Corresponding author. Tel.: +007 095 3247105; fax: .: +007 095 3247105; e-mail: boris@mail.cern.ch.
} 


\section{Silicon Photomultiplier description and performance}

\subsection{Principle of operation}

The Silicon Photomultiplier (SiPM) is a novel semi-conductor photodetector operated in limited Geiger mode [1]-[7]. It reaches an intrinsic gain for single photoelectrons at the level of $10^{6}$, comparable to that of vacuum phototubes (PMTs) and considerably higher than that of avalanche photodiodes (APDs) operated in proportional mode. The relatively inexpensive device consists of an array of small $(\sim 20-30 \mu \mathrm{m})$ independent pixels arranged on a common substrate to form a macroscopic unit of 1$3 \mathrm{~mm}$ size, with $500-4000$ pixels $/ \mathrm{mm}^{2}$. Each pixel is operated with a bias voltage $\mathrm{V}_{\text {bias }}$ a few volts above breakdown voltage $\mathrm{V}_{\text {breakdown. }}$ In this mode a photoelectron created in the silicon and reaching the high field region by diffusion or drift initiates a Geiger discharge confined to that pixel. The discharge is quenched by limiting the current to about $10 \mu \mathrm{A}$ with a small polysilicon resistor in each pixel. The independently operating pixels are connected to the same readout line; therefore the combined output signal corresponds to the sum of all fired pixels, which is a measure of the light flux

\subsection{Photon detection efficiency by SiPM}

Like other silicon-based photodetectors the SiPM has high quantum efficiency (QE) close to $100 \%$ for optical photons. However the overall photon detection efficiency (PDE) for present-state SiPMs is smaller due to several additional contributions apart from the QE:

- the geometrical efficiency $\varepsilon_{\text {geom }}$ (the fraction of sensitive area on the detector surface)

- the probability for a photo-electron to initiate a Geiger discharge

- the recovery time of the pixel

The spectral behaviour of the PDE is driven in general by the photon absorption length in $\mathrm{Si}$ (from 0.01 to few $\mu \mathrm{m}$ for wavelengths $300 \mathrm{~nm}<\lambda<700 \mathrm{~nm}$ ). Fig. 1 shows the PDE for present-state SiPMs in

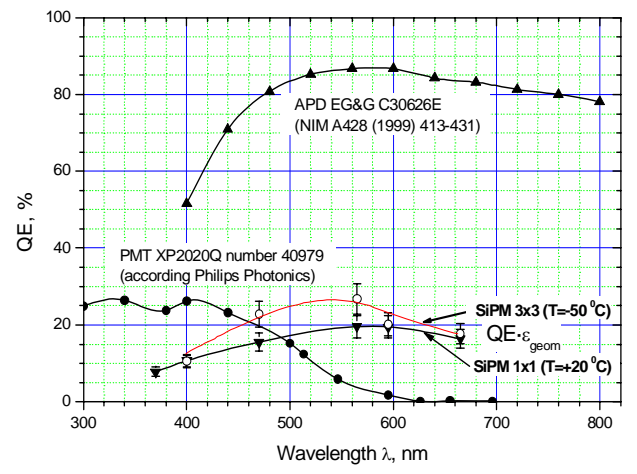

Fig. 1. Comparison of the Photon Detection Efficiency for SiPM, APD and PMT

comparison with the typical QE of APDs and PMTs. The SiPM PDE is at the level of the PMT QE for blue light and larger in the yellow-red region which is important for readout of scintillators via wavelength shifter (WLS) fibres.

The probability for a Geiger discharge (typically $60-70 \%$ ) and thus the overall SiPM PDE depend on the bias voltage through the ionization coefficient.

\subsection{Gain of SiPM}

The Geiger signal from one pixel is determined by the charge $\mathrm{Q}_{\text {pixel }}=\mathrm{C}_{\text {pixel }} \cdot \Delta \mathrm{V}$ accumulated in the pixel capacitance $\mathrm{C}_{\text {pixel. }}$. The overvoltage $\Delta \mathrm{V}=$ $\mathrm{V}_{\text {bias }}-\mathrm{V}_{\text {breackdown }}$.is of order of a few volts and $\mathrm{C}_{\text {pixel }}$ is typically $50 \mathrm{fF}$; so $\mathrm{Q}_{\text {pixel }}$ is of the order of $150 \mathrm{fC}$ or $10^{6}$ electrons. The single pixel gain increases linearly with overvoltage, in contrast to the exponential voltage dependence of the gain of APDs.

One pixel signal on $50 \mathrm{Ohm}$ load corresponds to a pulse amplitude of $\sim 1 \mathrm{mV}$ (for a typical 10-20 ns pulse) and can be transmitted to the front end electronics over several meters distance without requiring a preamplifier near the photodetector.

\subsection{Photon counting capability}

A SiPM pulse height spectrum generated by the dark rate is shown in Fig. 2. One can see an excellent single photoelectron (single pixel) resolution, which is a consequence of

- good pixel to pixel gain uniformity

- negligible contribution of electronics noise 
- a very low Excess Noise Factor (ENF) for each single pixel due to the Geiger mode operation, in contrast the large ENF for APDs due to fluctuations in the avalanche development process

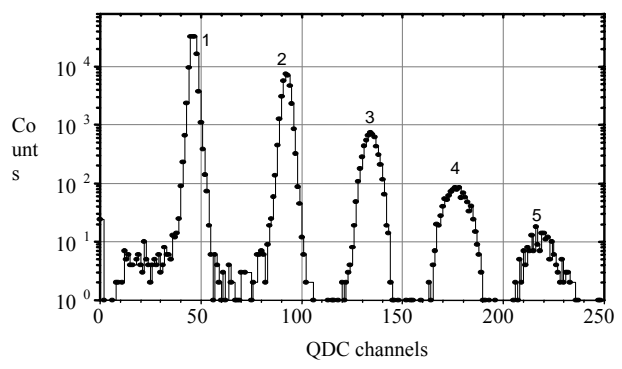

Fig. 2. Dark rate pulse height distribution.

The single photoelectron (single pixel) resolution is important for calibration and monitoring, because it allows to directly measure the SiPM gain via the distance between the maxima of pulse hight distribution.

\subsection{Single pixel dark rate}

The electronics noise of SiPMs is negligibly small due to the very high gain $\left(\sim 10^{6}\right)$, in contrast to standard APDs, where the gain is typically about 100 . In the SiPM the level of electronics noise corresponds to less than $10 \%$ of the signal from one photoelectron.

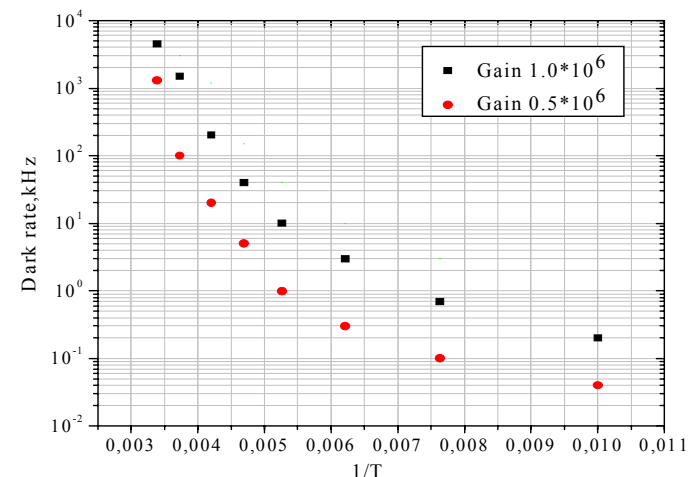

Fig. 3. The temperature dependence of the SiPM dark rate for different one pixel gains.
The main source of noise which limits the SiPM's single photon resolution is the dark rate, originating from charge carriers thermally created in the sensitive volume. The SiPM dark rate decreases with temperature from a few (typically $1-2$ ) $\mathrm{MHz} / \mathrm{mm}^{2}$ (room temperature) to $\sim 200 \mathrm{~Hz} / \mathrm{mm}^{2}$ (at $100 \mathrm{~K}$ ) - see Fig. 3.

For the detection of very small light signals (one or a few photoelectrons) on larger sensitive area this dark rate limits the SiPM performance at room temperatures. The rate can be reduced by operating at lower temperature, and by improving the SiPM production technology. The detection of large light signals is practically unaffected.

\subsection{Inter-pixel crosstalk}

The SiPM pixels should be decoupled from each other and work as independent photon microcounters. Electrical decoupling is realized by

- polysilicon quenching resistors for each pixel, which limit the Geiger discharge and at the same time electrically decouple pixel from pixel,

- specially designed boundaries between pixels in order to inhibit inter-pixel currents in the silicon itself. These boundaries occupy part of the SiPM surface and reduce the sensitive area (geometrical efficiency).

Another type of inter-pixel coupling is introduced by "optical crosstalk". It originates from photons created in the Geiger discharge with a rate of about $10^{-5}$ photons per electron [8]. These photons can propagate to another not primarily fired pixel and initiate a discharge there. Optical crosstalk violates the pixel independence and leads to a non-Poissonian behaviour of the distribution of the number of fired pixels. In Fig. 2, showing the dark rate pulse height distribution, one can see a long non-Poissonian tail., indicating an increased probability of multi-pixel firing.

For low dark rates (dark rate $\times$ SiPM pulse width $<<1$ ) an optical crosstalk coefficient $K_{2 / 1}$ can be defined as the probability for a second pixel to be fired. This coefficient (see Fig. 4) rises with the SiPM gain, since the number of secondary photons increases. The figure of merit is here the dark rate above a certain threshold. The dark rate dependence on gain, or bias voltage, is shown in Fig. 5. The 


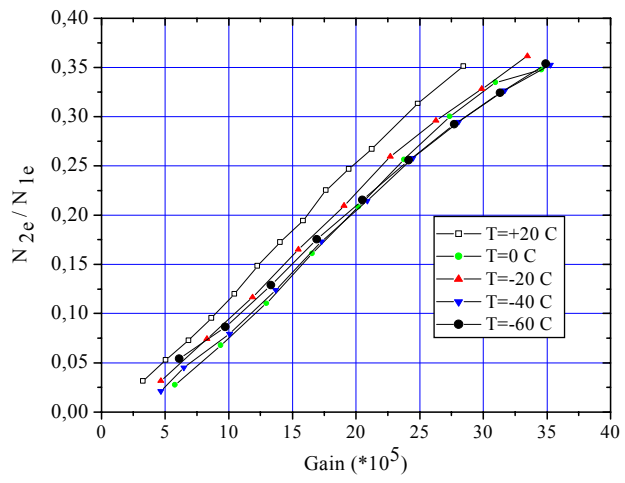

Fig. 4. Optical crosstalk coefficient (probability for second pixe to be fired compared a single pixel rate) vs SiPM one pixel gain.

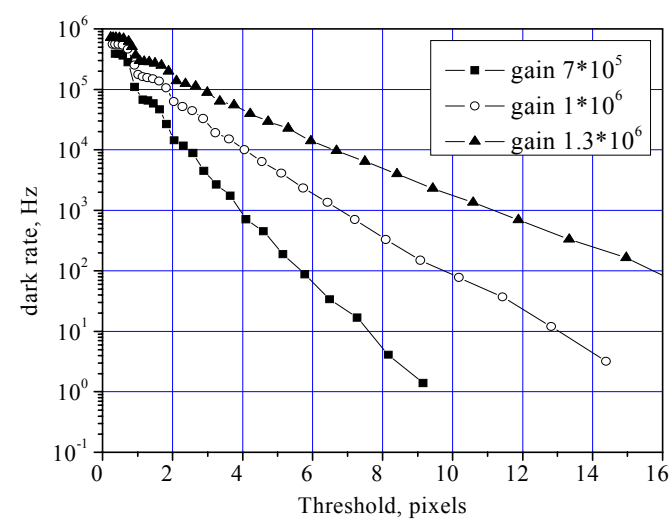

Fig. 5. The dark rate of the SiPM for different gains in dependence on the level of the threshold.

requirement to have a sufficiently low dark rate limits the gain for current SiPM's at the level of $10^{6}$, and the PDE to $10-15 \%$ (at room temperature).

\subsection{The timing by SiPM}

The SiPM response is intrinsically very fast (less than 500ps) due to the very thin depletion layer and the extremely short duration of the Geiger discharge development.

The single photoelectron timing resolution is characterized by $\mathrm{FWHM}_{0}=100$ ps ([2], [3]) for photons absorbed in the depletion region. The dependence of the timing resolution on $\mathrm{N}_{\text {photoelectrons }}$ as $\mathrm{FWHM}=\mathrm{FWHM}_{0} /\left(\mathrm{N}_{\text {photoelectrons }}\right)^{1 / 2}$ has been shown to be valid up to $\mathrm{N}_{\text {photoelectrons }}$ of 100 . It should be noted that such a single photoelectron timing resolution can be achieved using just a leading edge discriminator because the single photoelectron (single pixel) pulse is very stable (see Fig. 2).

The excellent timing properties of the SiPM can be useful for matching of the scintillation tile calorimeter signals in time to the bunch crossing of the collider with a resolution of about $1 \mathrm{~ns}$, which is determined by the rise time of scintillator + WLS fibre signal [9]. In the core of electromagnetic showers, with a signal corresponding to about 40 MIPs, a timing resolution of $\sim 200$ ps can be obtained.

The time for each pixel to recover from the discharge is also small. It is determined by the product of pixel capacity and polysilicon resistance, $\tau_{\mathrm{R}}=\mathrm{C}_{\text {pixel }} \cdot \mathrm{R}_{\mathrm{Si}}$. For polysilicon resistor values of $0.5-5$ $\mathrm{M} \Omega$ the recovery time of one pixel ranges between 25 and $250 \mathrm{~ns}$.

\subsection{SiPM dynamic range}

The finite number of SiPM pixels determines the dynamic range of the SiPM, and it leads to a nonlinearity of the SiPM response when the number of produced photoelectrons approaches the total number of pixels. Saturation occurs if the number of photoelectrons per pixel $\sim 1$. However, this is valid only if the duration of the light signal $\Delta \mathrm{t}$ is much smaller than the single pixel recovery time $\tau_{R}$. For long enough light signals $\left(\Delta t>\tau_{R}\right)$ the SiPM dynamic range is larger. 


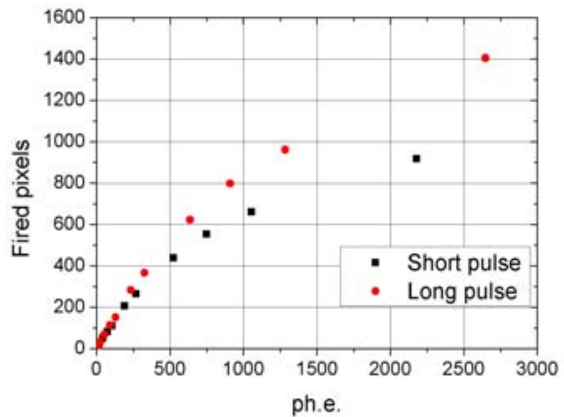

Fig. 6. Nonlinear response to LED light signals with different pulse durations.

On the other hand, in such a situation the nonlinear response function is strongly dependent on the width of the light pulse (see Fig. 6), which can complicate the nonlinearity correction procedure. A recovery time large compared to the light pulse duration would eliminate this potential problem; this would require the choice of a larger value for the polysilicon resistor.

\subsection{SiPM sensitivity to temperature and bias voltage}

The SiPM response to a given light signal depends on the product of photon detection efficiency and single pixel gain. Both factors depend on the overvoltage $\Delta \mathrm{V}(\mathrm{T})=\mathrm{V}_{\text {bias }}-\mathrm{V}_{\text {breackdown }}(\mathrm{T})$ and thus on the supplied bias voltage and via $\mathrm{V}_{\text {breackdown }}$ also on the temperature $\mathrm{T}$. The gain variation is $1.7 \% /{ }^{\circ} \mathrm{C}$ and $2.5 \% / 0.1 \mathrm{~V}$, and the overall SiPM signal amplitude variation is $4.5 \% /{ }^{\circ} \mathrm{C}$ and $7 \% / 0.1 \mathrm{~V}$ for a SiPM gain of $10^{6}$. The sensitivity to temperature and bias voltage decreases with increasing gain (and overvoltage).

\subsection{Calibration and monitoring of SiPMs}

The stability of the response of an individual scintillator + SiPM element can be monitored by using the position of the MIP or an $\alpha$-source signal. Fig. 7 demonstrates the possibility of such a monitoring concept for a $50 \mathrm{~mm} \times 50 \mathrm{~mm} \times 5 \mathrm{~mm}$ scintillator tile + WLS + SiPM system. It shows the single pixel spectrum obtained by using an LED, the
MIP signal (here from a $\mathrm{Sr}^{90} \beta$ source) and the signal generated by a $\mathrm{Pu}^{238} \alpha$-source. Because the plastic scintillator itself has very low light yield for $\alpha$ particles, a very small $(5 \mathrm{~mm} \times 5 \mathrm{~mm} \times 70 \mu \mathrm{m})$ YAP scintillator crystal has been glued on the centre of the plastic scintillator tile. The clear separation of the SiPM signal generated by a single particle provides a direct determination of the energy scale

$$
\frac{\text { energy deposition by MIP }}{\text { number of pixels fired }}
$$

for each scintillator element. The response measured in number of pixels per MIP must be known for corrections of the non-linear SiPM behaviour, since the SiPM dynamic range is determined by the total number of pixels.

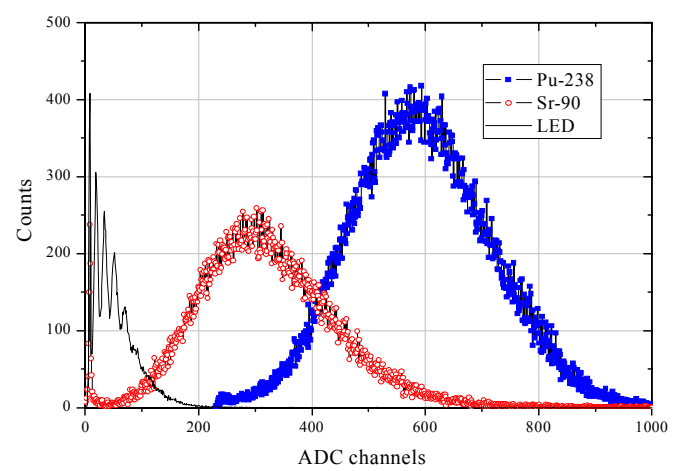

Fig. 7. The SiPM spectra for LED, beta-particles from $\mathrm{Sr}^{90}$ and alpha-particles from $\mathrm{Pu}^{238}$ source.

As already mentioned, the resolution of single pixel signals offers a unique possibility to perform a direct and absolute measurement of the SiPM gain and thus to monitor each SiPM for possible corrections due to variation of the temperature and bias voltage with time ("auto-calibration"). Using the known characteristics of the SiPM, the expected change in total response can be inferred from the observed change in gain. Direct gain measurement and radioactive sources thus provide alternatives to the more conventional method using a stabilized light source as reference signal. 


\subsection{Low bias voltage, low power consumption}

The breakdown electric field in $\mathrm{Si}$ is about $2.5 \cdot 10^{5}$ $\mathrm{V} / \mathrm{cm}$. Since the SiPM depletion zone is typically 1 to $3 \mu \mathrm{m}$ thin, the working bias voltage is rather low (25 to $75 \mathrm{~V})$. This is comfortable for the design of systems with a large number of SiPM's. For instance, a single coax cable is needed to supply bias voltage and provide the signal line to one SiPM, and one can even use the cable screen as bias voltage line.

The typical dark current for a $1 \mathrm{~mm}^{2} \mathrm{SiPM}$ is about $1 \mu \mathrm{A}$, therefore the corresponding power consumption is also very small $(\sim 50 \mu \mathrm{W})$.

\subsection{SiPM compactness}

The SiPM device is very compact: for $1 \mathrm{~mm} \times 1 \mathrm{~mm}$ sensitive area the total SiPM size is as small as $1.5 \mathrm{~mm} \times 1.5 \mathrm{~mm}$ with a thickness of $300 \mu \mathrm{m}$ of $\mathrm{Si}$. Together with the low bias voltage and power consumption, this opens up new possibilities for the readout of scintillator elements: to mount the SiPM directly embedding the photodetector into the body of the scintillator (see Fig 8).

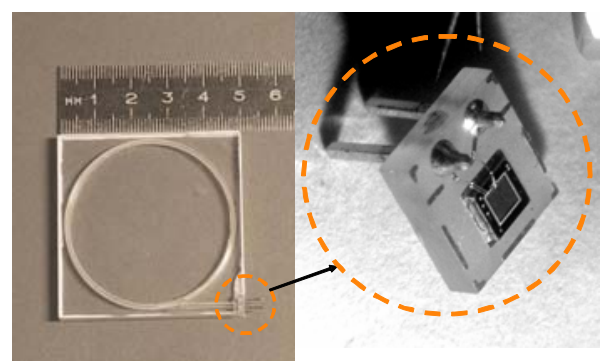

Fig. 8. The SiPM mounted directly on tile.

\subsection{SiPM: very low charge particle sensitivity (negligible nuclear counting effect)}

The SiPM signal does not depend on the number of primary carriers created (because of the Geiger mode operation). Thus, each pixel detects the carriers created by a photon, by a minimum ionizing particle, or by thermal excitation with the same response signal of $\sim 10^{6}$ electrons. Together with the compactness, this implies that the nuclear counter effect is negligible for SiPMs, and they can be located inside the calorimeter, for example.

\subsection{Insensitivity to magnetic field}

Most calorimeters for collider detectors operate in high magnetic fields. The operation of SiPMs has been tested in a magnetic field up to $4 \mathrm{~T}$ for two orientations of the field lines [10] and it was shown that the SiPM signal is stable within $1 \%$ measurement accuracy. Furthermore, dark current, noise frequency, gain and pixel crosstalk of SiPM have been measured at $4 \mathrm{~T}$ and found to be magnetic field independent.

\subsection{Large area SiPM's [11]}

There are some limitations for the performance of large area, high gain and high photon detection efficiency SiPMs:

- the dark rate, which increases proportionally with area.

- power dissipation

- optical crosstalk

All these reasons are more and more significant for larger area SiPM with high packing efficiency of the pixels and limit the SiPM size at the level of $10 \times 10 \mathrm{~mm}^{2}$ for moderate cooling $\left(-30 \ldots-50{ }^{\circ} \mathrm{C}\right)$. Nevertheless the SiPM with area of $3 \times 3 \mathrm{~mm}^{2}$ has been successfully built and operated [11] - see Fig. 9 and Fig. 1, where its photon detection efficiency is shown.

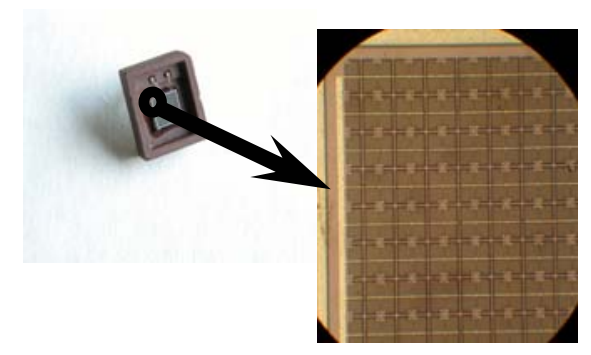

Fig. 9. SiPM $3 \times 3 \mathrm{~mm}^{2}, 5625$ pixels. 


\subsection{SiPM long term stability and ageing}

Two dedicated tests have been performed in order to study the SiPM long term stability and ageing under different conditions.

First, a sample consisting of $20 \mathrm{SiPMs}(1 \mathrm{~mm} \times 1$ $\mathrm{mm}, 576$ pixels) was operated under bias voltage corresponding to a gain of $1 \times 10^{6}$ during 1500 hours at room temperature. The SiPM parameters such as gain, photon detection efficiency, dark noise rate and dark current were measured before the test, after 500 hours and after 1500 hours of operation.

Second, possible ageing of 5 SiPMs with 1024 pixels was studied under extreme temperature conditions ranging from room temperature up to $90^{\circ} \mathrm{C}$, increased in steps of $10^{\circ} \mathrm{C}$. At each temperature point the SiPMs were operated for 24 hours with a gain of $1 \times 10^{6}$ (with increasing temperature the bias voltage was increased, too). The SiPM parameters did not show any variation during these stability tests. More long term SiPM stability studies including the radiation resistance are needed and planed in nearest future.

\section{Some examples of SiPM applications}

\subsection{Scintillator $+W L S+S i P M$ systems}

\subsubsection{High granularity Sci Tile Hadron Calorimeter prototypes for International Linear $e^{+} e^{-}$Collider Detector [10, 12, 13]}

The precision physics program at a future Linear Collider (LC) requires reconstructing hadronic final states of heavy boson (W, Z, H) decays in multi-jet events. For this purpose a jet energy resolution of better than $30 \% / \sqrt{ } \mathrm{E}$ is necessary. Monte Carlo simulations demonstrate that such a resolution can be achieved using a novel "particle flow" approach in which each particle in a jet is measured individually. In this approach the calorimeter must have very fine longitudinal and transverse segmentation. For hadron shower separation a transversal segmentation of about $3 \times 3 \mathrm{~cm}^{2}$ is needed, and every sampling layer should be read out individually. Such a granularity can be realized on large scale using scintillator tiles with wave length shifter (WLS) fibre light collection, individually read out by Silicon photo-multiplier (SiPM) as photodetectors.

A first prototype calorimeter based on scintillator tiles of $5 \mathrm{~cm} \times 5 \mathrm{~cm} \times 5 \mathrm{~mm}$ size (Fig. 8) with 108 channels ("minical") has been constructed. The light yield of the tiles produced a signal corresponding to 25- 4 fired pixels per MIP. The calorimeter design, data taking, calibration and results are reported in [10].

This "minical" has been successfully operated in the DESY positron test beam at energies of from 1 to $6 \mathrm{GeV}$. Nonlinearity effects due to the limited number of pixels were noticeable in the shower core; for $6 \mathrm{GeV}$ electromagnetic showers the energy depositions were ranging up to an equivalent of 70 MIPs per tile). The effects could successfully be corrected for each channel using the well-measured SiPM response function. Fig. 10 shows the measured energy resolution as a function of beam energy. The data compare well with results obtained with conventional multi-anode vacuum phototubes and they are well reproduced by a Monte Carlo simulation which includes the detailed SiPM behaviour. This leads to conclusion that after correction the saturation effects do not degrade the calorimeter linearity and resolution.

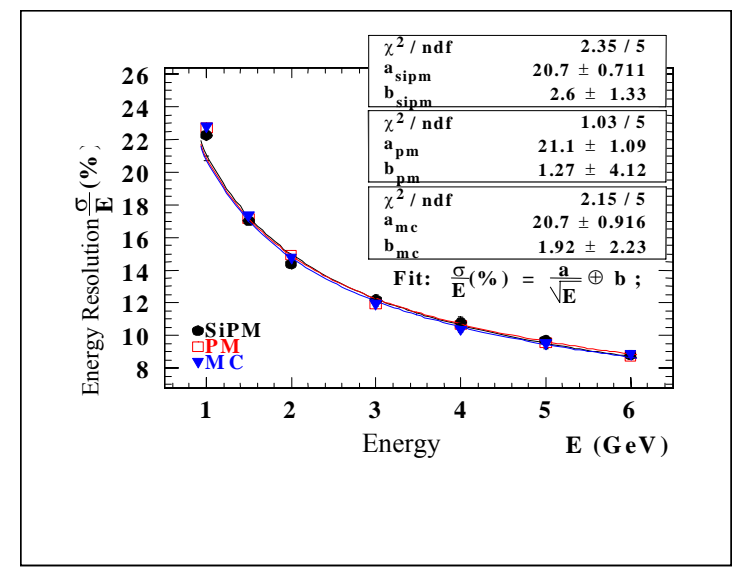

Fig. 10. Measured energy resolution for SiPM with (solid points), MAPM (squares) and MC prediction (triangles).

The next step in the development of a high granularity scintillator tile hadron calorimeter is the 
$1 \mathrm{~m}^{3}$ prototype $[12,13]$ which is being constructed by the CALICE collaboration and consists of 40 layers of $3 \times 3,6 \times 6$ and $12 \times 12 \mathrm{~cm}^{2} 0.5 \mathrm{~cm}$ thick tiles + WLS fibers + SiPMs.. Fig. 11 shows one layer of this prototype under assembly. The total number of channels is 8000 and requires a significant advancement in mass production and quality control.

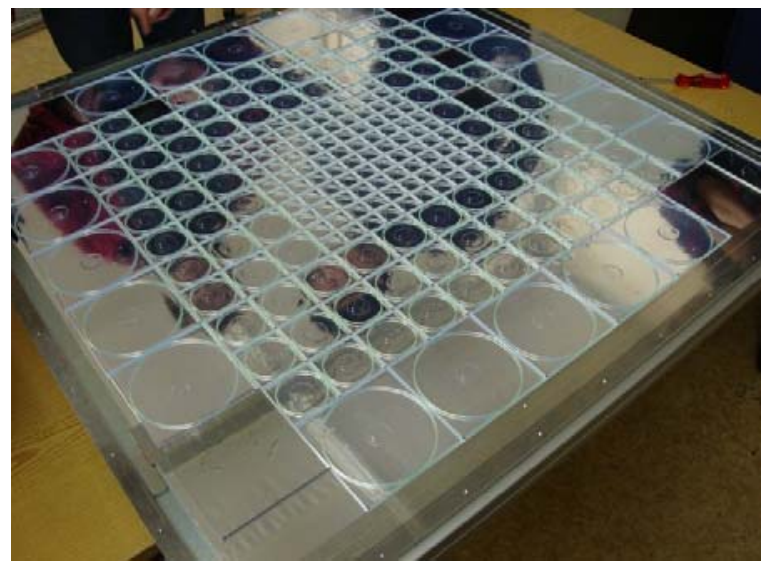

Fig. 11. One plane with SiPMs and WLS fibers installed into $3 \times 3$, $6 \times 6$ and $12 \times 12 \mathrm{~cm}^{2} 0.5 \mathrm{~cm}$ thick tiles.

\subsubsection{Scintillator based hodoscope with SiPM's.}

Fig. 12 shows an example of such a scintillator $+\mathrm{WLS}+\mathrm{SiPM}$ system for $2 \mathrm{~m} \times 25 \mathrm{~mm} \times 10 \mathrm{~mm} \operatorname{rod}[14]$ : an efficiency for MIP detection of more than $99 \%$ has been achieved even with a SiPM on one end only.

A $(5 \mathrm{~cm} \times 5 \mathrm{~cm} \times 5 \mathrm{~mm})$ hodoscope scintillator tile + WLS + SiPM prepared by MEPHI-ITEP has been successfully operated within the space experiment LAZ10 (MEPHI-INFN collaboration) on the International Space Station (launched on $15^{\text {th }}$ of April, 2005) for the measurement of low energy particle fluxes and radiation monitoring. Fig. 13 displays the latitude particle flux dependence measured by this space SiPM based hodoscope.

Another example of such a hodoscope is the $8 \mathrm{~m}^{2}$ Alice TOF Cosmic Test System [15] with scintillator

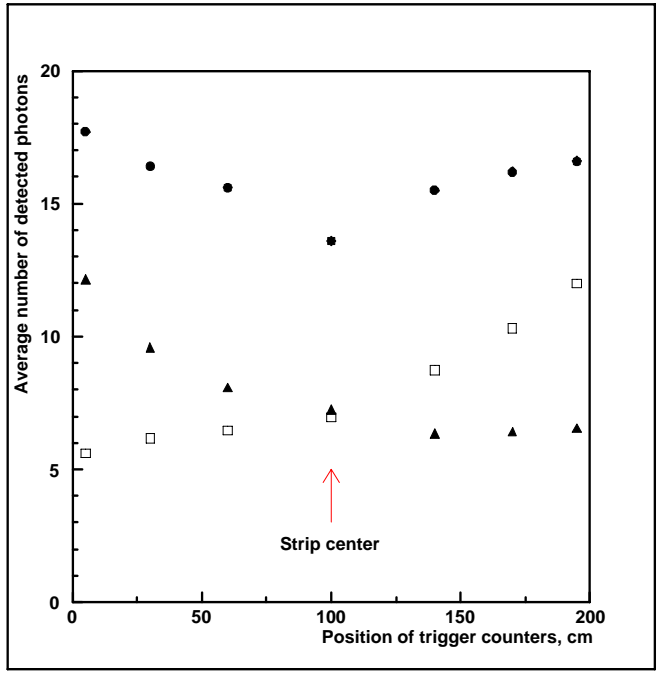

Fig. 12. SiPM pulse height in number of photoelectrons vs MIP particle coordinate along the scintillation bar $(2.5 \mathrm{~cm} \times 1 \mathrm{~cm} \times 2 \mathrm{~m})$. Lower curves correspond the individual SiPM from both ends of the bar, upper one is a sum of both SiPM's.

tiles of $15 \times 15 \times 1 \mathrm{~cm}^{3}$ size. ${ }^{*}$;A timing resolution of $\sim 1.2$ ns has been obtained.

\subsection{SiPM application for scintillating fiber MIP detection}

Fig. 14 shows the pulse height spectrum from MIP detection by (Kuraray SCSF-78M) $1 \mathrm{~mm}$ diameter scintillating fiber $+\mathrm{SiPM}$ at room temperature. One can see the Landau-like distribution with clearly separated photoelectron (fired pixel) peaks $(\sim 7$ pixels/MIP).

\subsection{SiPM usage for Liquid Xe scintillation detection}

This application confirms the possibility of vacuum UV light detection by SiPMs in experiments on dark matter WIMPS search [16]. The scintillation light in liquid $\mathrm{Xe}\left(-95{ }^{\circ} \mathrm{C}\right)$ has a wavelength of $178 \mathrm{~nm}$, The pulse hight spectrum of $\mathrm{Am}^{241}$ alpha particles is shown in Fig. 15; a photon detection

\footnotetext{
*) This hodoscope used the Geiger mode APD called "MRS APD" (which is quite similar to SiPM), produced by the Centre of Perspective Technology and Apparatus, Moscow.
} 

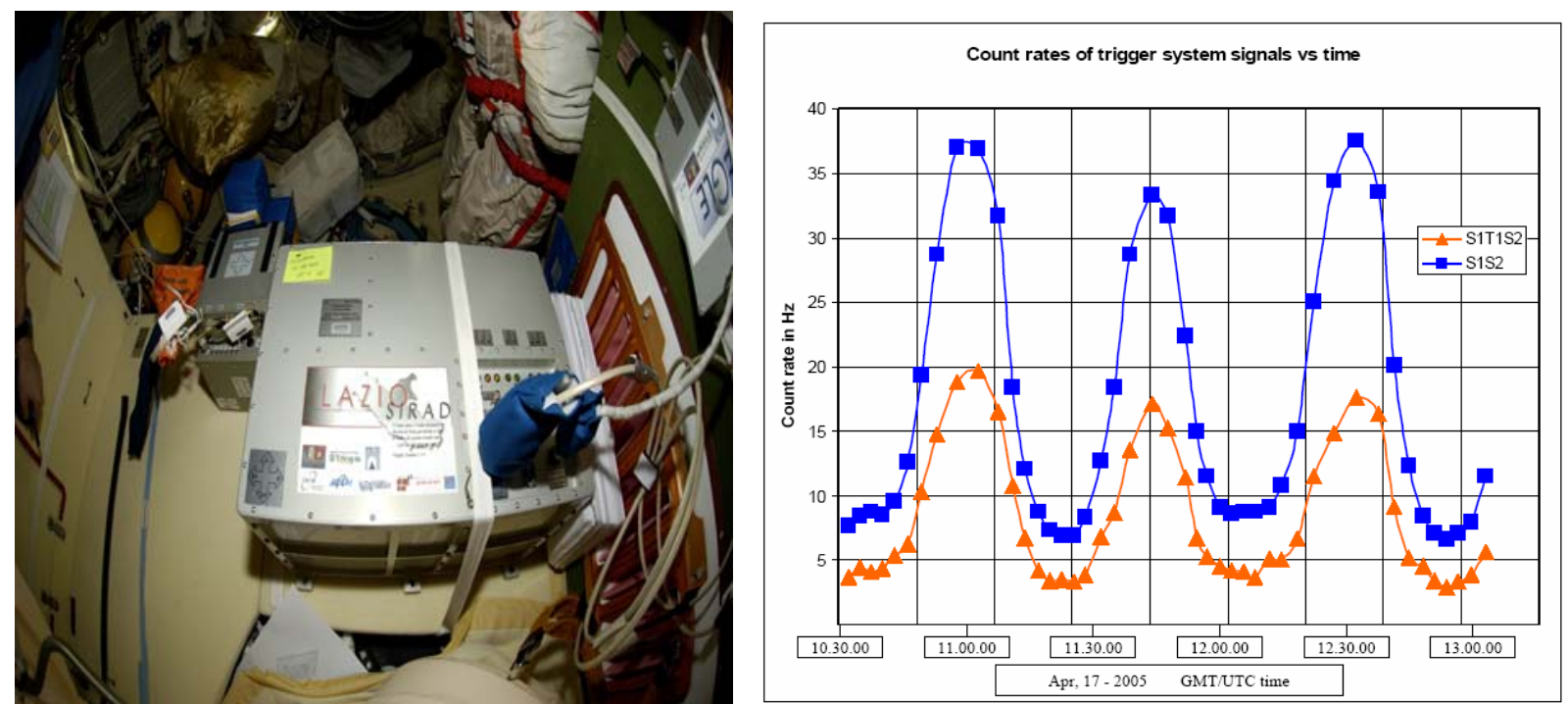

Fig. 13. Sci+WLS fiber+SiPM hodoscope system in space experiment "LAZ10".

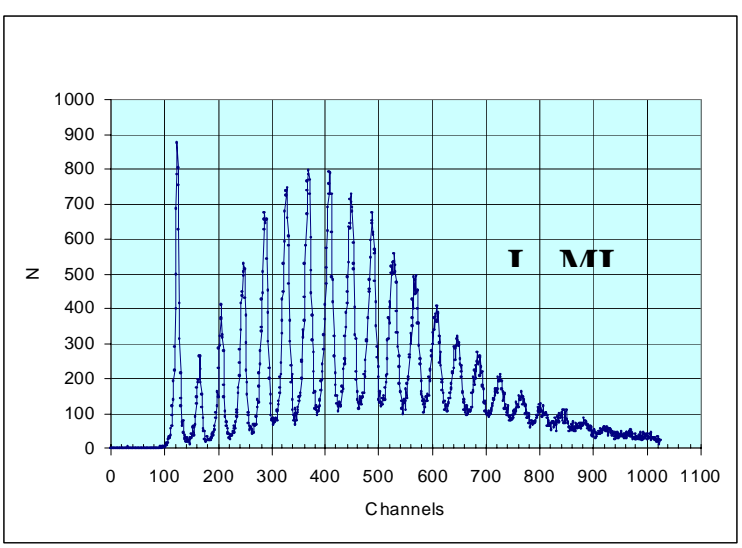

Fig. 14. MIP detection by sci fiber+SiPM.

efficiency of the SiPM $(1 \mathrm{~mm} \times 1 \mathrm{~mm}, 576$ pixels $)$ of $5.5 \%$ for $\lambda=178 \mathrm{~nm}$ has been measured.

\subsection{A few examples of larger area $\left(3 \times 3 \mathrm{~mm}^{2}\right)$ SiPM's applications}

\subsubsection{Transition $X$-ray detection}

Transition Radiation X-ray detectors based on very thin heavy scintillator+SiPM appear to be more

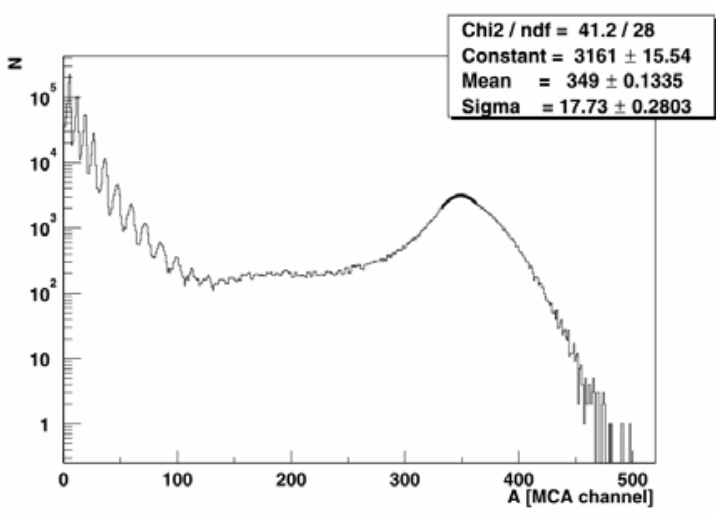

Fig. 15. Am ${ }^{241}$ alpha-particle scintillations in LXe, $178 \mathrm{~nm},-95^{\circ} \mathrm{C}$ ).

robust and fast than traditional Xe gas based TRDs, especially for space physics applications (no gas, no high voltage). Fig. 16 shows the results from a test prototype [11] based on $3 \times 3 \mathrm{~mm}^{2} \mathrm{SiPM}+70 \mu \mathrm{m}$ YAP:Ce crystal. Such a system is a promising candidate for a base element of multiset TR detectors. 

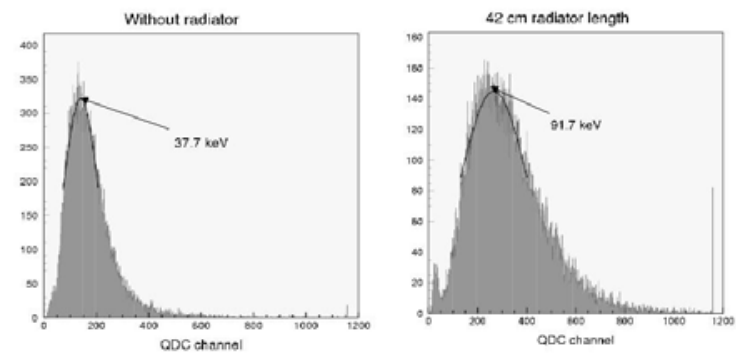

Fig. 16. Pulse height distributions for TR X-ray detection with one polypropylen foils radiator and YAP:Ce crystal $+3 \times 3 \mathrm{~mm}^{2}$ SiPM.

\subsubsection{Time of Flight measurements with SiPM [9,} 11]

The timing resolution of a system consisting of a $3 \times 3 \mathrm{~mm}^{2} \mathrm{SiPM}+$ plastic scintillator BC418 $(3 \times 3 \times 40$ $\mathrm{mm}^{3}$, decay time $1.4 \mathrm{~ns}$ ) has been measured for $3 \mathrm{GeV}$ electrons to $\sigma(\mathrm{SiPM}+\mathrm{BC} 418)=32 \mathrm{ps}$. Such a result confirms the high potential of TOF systems based on large area $\mathrm{SiPM}+$ plastic scintillator elements.

\subsubsection{SiPM application for Positron Emission Tomography (PET)}

Fig. 17 demonstrates the possibility for detection of PET photons $(511 \mathrm{keV})$ by an SiPM $\left(3 \times 3 \mathrm{~mm}^{2}\right)$ coupled to a LYSO crystal $\left(3 \times 3 \times 20 \mathrm{~mm}^{3}\right)$. A pulse height resolution of $17.5 \%(\mathrm{FWHM})$ and a timing

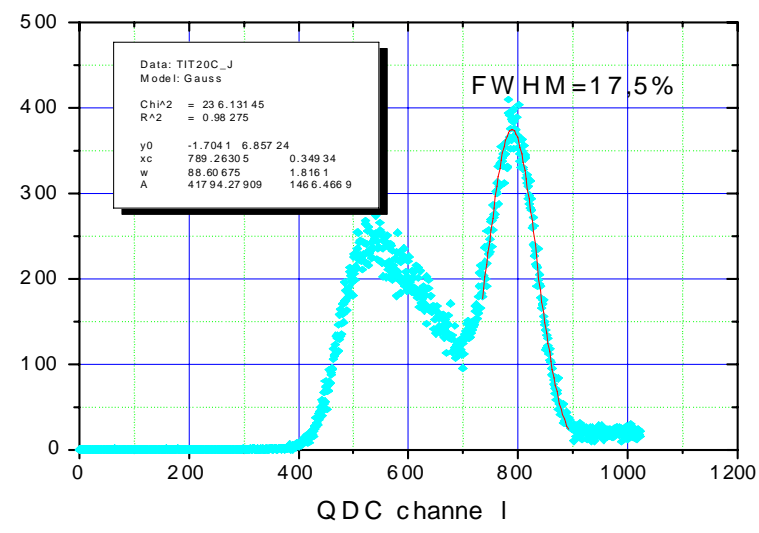

Fig. 17. Pulse height distribution for $511 \mathrm{keV}$ gamma`s, obtained by LYSO $\left(3 \times 3 \times 20 \mathrm{~mm}^{3}\right)+3 \times 3 \mathrm{~mm}^{2} \mathrm{SiPM}$ system. resolution between two PET photons of 780 ps have been measured $[9,11]$. Low power consumption, low voltage, excellent timing and compactness give rise to a high potential for SiPMs to be used for new generation of PET tomographs $[11,17]$.

\section{Conclusions}

The Silicon Photomultiplier is becoming a photodetection device competitive to PMT and APD for a number of applications. A new generation of SiPMs with reduced

- optical crosstalk

- dark rate

- afterpulsing

is under development now in order to obtain SiPMs with - area up to $10 \times 10 \mathrm{~mm}^{2}$

- photon detection efficiency of 40-50\%

- excess noise factor as low as a few \%

- subnanosecond timing

It will significantly increase the number of SiPM applications even further.

\section{References}

[1] G. Bondarenko, B. Dolgoshein, V. Golovin, A. Ilyin, R. Klanner, E. Popova, Nucl. Phys. B (Proc. Suppl.), 61 B (1998) 347 and reference therein,G. Bondarenko, P. Buzhan, B. Dolgoshein, V. Golovin, E. Guschin, A. Ilyin, V. Kaplin, A. Karakash, R. Klanner, V. Pokachalov, E. Popova, K. Smirnov,'Limited Geiger-mode microcell silicon photodiode: new results," Nucl. Instrum. Methods, vol. A442, pp. 187-192, 2000 and references therein

[2] P. Buzhan, B. Dolgoshein, L. Filatov, A. Ilyin, V. Kantzerov, V. Kaplin, A. Karakash, F. Kayumov, S. Klemin, A. Pleshko, E. Popova, S. Smirnov, Yu. Volkov, "The Advanced Study of Silicon Photomultiplier," Proceedings of the 7th International Conference on Advance Technology \& Particle Physics, 2002, pp. 717-728.

[3] P. Buzhan, B. Dolgoshein, L. Filatov, A. Ilyin, V. Kantzerov, V. Kaplin, A. Karakash, F. P. Buzhan, B. Dolgoshein, L. Filatov, A. Ilyin, V. Kantzerov, V. Kaplin, A. Karakash, F. Kayumov, S. Klemin, E. Popova, S. Smirnov, "Silicon photomultiplier and its possible applications," Nucl. Instrum. Methods, vol. A504, pp. 48-52, 2003.

[4] B. Dolgoshein (on behalf of SiPM-Collaboration), "Silicon photomultipliers in Particle Physics: possibilities and limitations," presented at Workshop "Innovative detectors for future Colliders", Erice, Italy, Oct. 2003. 
[5] D. Renker, talk given at Beaune-2005 Conference and references therein, Proceedings of 4th International Conference "New Development in Photodetection", June 2005, Beaune, submitted to NIMA.

[6] Yu. Musienko, contribution given at Beaune-2005 Conference, Proceedings of 4th International Conference "New Development in Photodetection", June 2005, Beaune, submitted to NIMA.

[7] Z. Sadygov, talk given at Beaune-2005 Conference and references therein, Proceedings of 4th International Conference "New Development in Photodetection", June 2005, Beaune, submitted to NIMA.

[8] Lacaita, F. Zappa, S. Bigliardi, M. Manfredi, "On the Bremsstrahlung Origin of Hot-Carrier-Induced Photons in Silicon Divices," IEEE Trans. Electron Devices, vol. 40, no. 3, pp. 577-582, March 1993

[9] Karakash, contribution given at Beaune-2005 Conference, Proceedings of 4th International Conference "New Development in Photodetection”, June 2005, Beaune, submitted to NIMA.

[10] V. Andreev, V. Balagura, B. Bobchenko, P. Buzhan, J. Cvach, M. Danilov et al. "A high granularity scintillator hadroniccalorimeter with SiPM readout for a linear collider detector", NIMA A540 (2005) 368.

[11] Dolgoshein, talk given at Beaune-2005 Conference, Proceedings of 4th International Conference "New Development in Photodetection”, June 2005, Beaune, submitted to NIMA.

[12] M. Danilov, talk given at Lepton-Photon Simposium, 2005; F.Sefkow, proceedings of LCWS2005, Stanford, USA, 2005.

[13] E. Popova, talk given at Beaune-2005 Conference, Proceedings of 4th International Conference "New Development in Photodetection”, June 2005, Beaune, submitted to NIMA.

[14] V. Balagura et al. "Study of Scintillator Strip with Wavelength Shifting Fiber and Silicon Photomultiplier". It is submitted to NIM.

[15] Martemyanov, talk given at Beaune-2005 Conference, Proceedings of 4th International Conference "New Development in Photodetection", June 2005, Beaune, submitted to NIMA.

[16] E. Aprile, P. Cushman, K. Nu, P. Shagin, submitted to NIMA

[17] A.N. Otte at al. NIMA 545(2005), 705 